\title{
NGC 1277: A MASSIVE COMPACT RELIC GALAXY IN THE NEARBY UNIVERSE
}

\author{
Ignacio Trujillo ${ }^{1,2}$, Anna Ferré-Mated ${ }^{3}$, Marc Balcells ${ }^{4}$, AleXandre Vazdekis $^{1,2}$, And Patricia Sánchez-BlázQuez ${ }^{5}$ \\ ${ }^{1}$ Instituto de Astrofísica de Canarias, c/Vía Láctea s/n, E-38205-La Laguna, Tenerife, Spain; trujillo@iac.es \\ ${ }^{2}$ Departamento de Astrofísica, Universidad de La Laguna, E-38205 La Laguna, Tenerife, Spain \\ ${ }^{3}$ Subaru Telescope, 650 North A'ohoku Place, Hilo, HI 96720, USA \\ ${ }^{4}$ Isaac Newton Group of Telescopes, E-38700 Santa Cruz de La Palma, Canary Islands, Spain \\ ${ }^{5}$ Departamento de Física Teórica, Universidad Autónoma de Madrid, E-28049, Cantoblanco, Madrid, Spain \\ Received 2013 October 23; accepted 2013 November 26; published 2013 December 16
}

\begin{abstract}
As early as $10 \mathrm{Gyr}$ ago, galaxies with more than $10^{11} M_{\odot}$ of stars already existed. While most of these massive galaxies must have subsequently transformed through on-going star formation and mergers with other galaxies, a small fraction $(\lesssim 0.1 \%)$ may have survived untouched until today. Searches for such relic galaxies, useful windows to explore the early universe, have been inconclusive to date: galaxies with masses and sizes like those observed at high redshift $\left(M_{\star} \gtrsim 10^{11} M_{\odot} ; R_{e} \lesssim 1.5 \mathrm{kpc}\right)$ have been found in the local universe, but their stars are far too young for the galaxy to be a relic galaxy. This paper explores the first case of a nearby galaxy, NGC 1277 (at a distance of $73 \mathrm{Mpc}$ in the Perseus galaxy cluster), which fulfills many criteria to be considered a relic galaxy. Using deep optical spectroscopy, we derive the star formation history along the structure of the galaxy: the stellar populations are uniformly old $(>10 \mathrm{Gyr})$ with no evidence for more recent star formation episodes. The metallicity of their stars is super-solar $([\mathrm{Fe} / \mathrm{H}]=0.20 \pm 0.04$ with a smooth decline toward the outer regions $)$ and $\alpha$-enriched $([\alpha / \mathrm{Fe}]=$ $0.4 \pm 0.1$ ). This suggests a very short formation time scale for the bulk of the stars in this galaxy. This object also rotates very fast $\left(V_{\text {rot }} \sim 300 \mathrm{~km} \mathrm{~s}^{-1}\right)$ and has a large central velocity dispersion $\left(\sigma>300 \mathrm{~km} \mathrm{~s}^{-1}\right)$. NGC 1277 allows the exploration in full detail of properties such as the structure, internal dynamics, metallicity, and initial mass function as they were at $\sim 10-12$ Gyr ago when the first massive galaxies were built.
\end{abstract}

Key words: galaxies: elliptical and lenticular, $\mathrm{cD}$ - galaxies: evolution - galaxies: formation - galaxies: photometry - galaxies: structure

Online-only material: color figures

\section{INTRODUCTION}

We define a relic galaxy as an object that was formed in the early phases of the universe (i.e., $z>2$ ) and which has remained unaltered (i.e., without significant gas or stellar accretion) since its initial formation. Finding an object with these characteristics in the local universe would allow a detailed study of its assembly history and significantly advance our understanding of the first steps in galaxy formation. The question is thus how to identify one of these objects in our neighborhood. One obvious characteristic that these relic galaxies should possess is that they must be old along their entire structure with no sign of star formation after galaxy formation. In other words, their star formation history ( $\mathrm{SFH}$ ) should peak at the beginning of the universe and not show any activity later on.

Among present-day galaxies, the most promising candidates for relics are the massive ellipticals. Their SFHs are compatible with little or negligible star formation (e.g., Vazdekis et al. 1997) after their initial formation. However, their structures are very different (i.e., their sizes have grown by a factor of $\sim 4$; Trujillo et al. 2007) compared to the size of their likely progenitors at $z>2$, implying that they have assembled a significant fraction of their stars in the last 10 Gyr. For this reason, if we want to find present-day galaxies which have remained unaltered since their initial formation at $z>2$, we have to search for current old, massive galaxies which have the same structural properties as the population of massive galaxies at high- $z$. That is, finding old (>10 Gyr), massive $\left(M_{\star} \gtrsim 10^{11} M_{\odot}\right)$, and compact $\left(R_{e} \lesssim 1.5 \mathrm{kpc}\right)$ galaxies in our vicinity.

Recently, Quilis \& Trujillo (2013) estimated the number density and the expected fraction of massive relic galaxies in our nearby $(z \lesssim 0.1$ ) universe. They found that only $\sim 0.1 \%$ of current massive galaxies are expected to have accreted less than $\sim 10 \%$ of their stars after their formation. This implies that the number density of massive relics today should be $\sim 10^{-6} \mathrm{Mpc}^{-3}$. These figures are small, but thanks to large area surveys like the Sloan Digital Sky Survey (SDSS) we should be able to find $\sim 60$ massive relics down to $z=0.1$ (in the $8032 \mathrm{deg}^{2}$ covered by the spectroscopic SDSS Legacy DR7). Searches for massive compact galaxies in the nearby universe have found some (although fewer than expected) candidates for relic objects (e.g., Trujillo et al. 2009; Valentinuzzi et al. 2010; Taylor et al. 2010; Poggianti et al. 2013) but it became apparent that these galaxies are surprisingly young $(\sim 2 \mathrm{Gyr}$; Ferré-Mateu et al. 2012). It is unclear whether the absence of relics in the SDSS Legacy footprint could be related to spectroscopic incompleteness in some particular areas of the sky. For instance, in rich galaxy clusters, the spatial proximity of the galaxies could have prevented proper spectroscopic coverage of the targets.

The object that we explore in this Letter, NGC 1277, is located at $73 \mathrm{Mpc}$ in the Perseus galaxy cluster. This cluster is outside the SDSS DR7 Legacy coverage. However, this field was targeted by an exploratory survey in preparing the SDSS SEGUE program. NGC 1277 has recently received a lot of attention due to the claim by van den Bosch et al. (2012) that this object has an extraordinary large supermassive black hole (SMBH). Interestingly, NGC 1277 is massive $\left(M_{\star}=1.2 \pm\right.$ $\left.0.4 \times 10^{11} M_{\odot}\right)$ and compact $\left(R_{e}=1.2 \mathrm{kpc}\right.$ ), two of the three properties we require for a massive galaxy to be a relic. This Letter explores the third condition for relic galaxies and presents a detailed analysis of the SFH of NGC 1277 along its global structure. The SDSS spectrum of this galaxy is insufficient for 


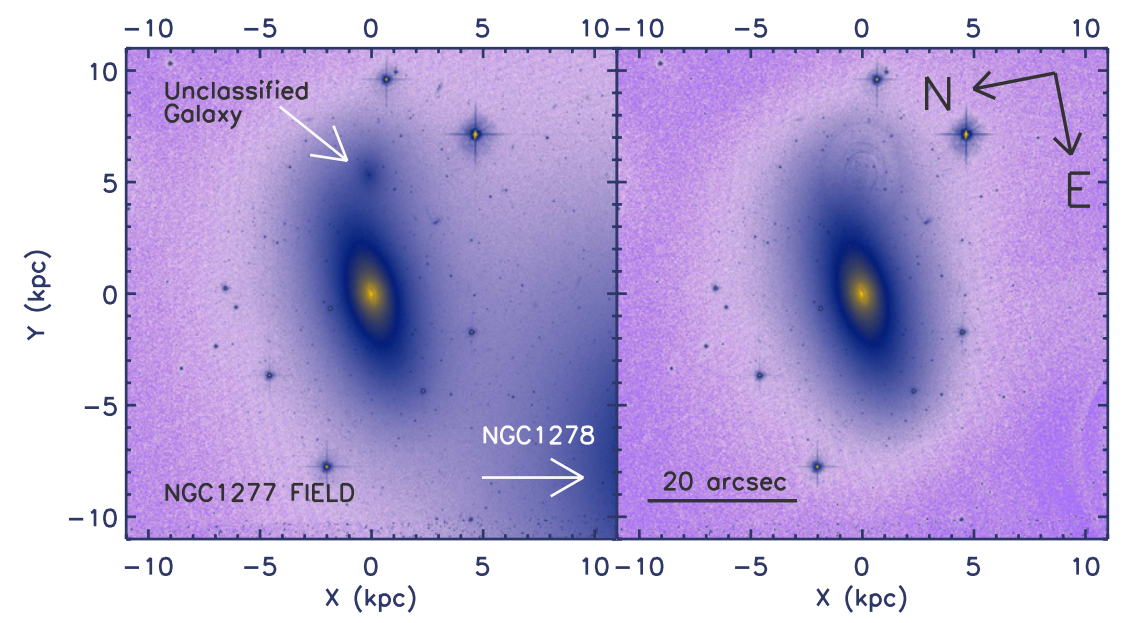

Figure 1. Neighborhood of NGC 1277 as seen by the HST F625W filter. The left panel shows the two closest galaxies whose light contaminate NGC 1277. The right panel shows NGC 1277 after the subtraction of the contaminant light. The results indicates that NGC 1277 is rather symmetric with no distortions or bright tidal streams surrounding it.

(A color version of this figure is available in the online journal.)

this purpose as it only covers its inner $0.5 \mathrm{kpc}$. We use our own very deep spectroscopy to perform a stellar population analysis out to $\sim 3 R_{\mathrm{e}}$. NGC 1277 is uniformly old, which makes it a strong candidate for a relic galaxy.

Throughout this Letter we adopt a standard cosmological model with the following parameters: $H_{0}=70 \mathrm{~km} \mathrm{~s}^{-1} \mathrm{Mpc}^{-1}$, $\Omega_{m}=0.3$, and $\Omega_{\Lambda}=0.7$. The redshift adopted here for NGC $1277, z=0.0169$, corresponds to a galaxy distance of $73.3 \mathrm{Mpc}$ and to a spatial scale of $344 \mathrm{pc} \operatorname{arcsec}^{-1}$.

\section{DATA}

\subsection{Hubble Space Telescope Imaging}

The stellar mass density profile of NGC 1277 as well as high resolution imaging of the galaxy have been obtained using the WFC Advanced Camera for Surveys F625W (Sloan $r$ ) filter from the NASA/ESA Hubble Space Telescope (HST) archive. These observations are associated with the program GO:10546 (PI: Fabian). The pixel scale of this image is 0'05 and the pointspread function FWHM is $0{ }^{\prime} 1$. The photometric zeropoint is 25.893 (AB system). Figure 1 shows an image of this galaxy in the F625W band.

\subsection{Deep Long-slit Spectroscopy}

We obtained deep long-slit spectra employing the ISIS spectrograph on the $4.2 \mathrm{~m}$ William Herschel Telescope. The blue grating $\mathrm{R} 300 \mathrm{~B}$ was centered at $5300 \AA$ with a $\times 2$ binning in the spatial direction and the $1^{\prime \prime}$ slit was placed along the galaxy's major axis $\left(\right.$ P.A. $\left.=96^{\circ}\right)$. This setup provided a spectral resolution of $3.4 \AA$. Seeing was 0.7 during the observations. Six exposures of 30 minutes were taken, giving a total time on source of $3 \mathrm{hr}$. Two spectrophotometric stars were observed with the same configuration to correct the shape of the spectra.

Data reduction was performed with REDUCEME (Cardiel 1999), a package optimized for long-slit spectroscopy that allows a parallel treatment of the scientific data and the errors propagated through the process. This reduction process included a bias subtraction, flat-fielding, cosmic-ray removal, C- and S-distortion correction, wavelength calibration, sky subtraction, and flux calibration. The result is a high quality spectrum (averaged central signal-to-noise ratio $(\mathrm{S} / \mathrm{N})>150)$ that covers the wavelength range $\lambda \lambda 3400-6200 \AA$. This coverage is optimal as it encompasses most of the line-strength indices employed in stellar population analysis and is also wide enough for the full spectral fitting technique in which this exercise is mostly based on.

\section{ANALYSIS}

One of the conditions that we require for a relic galaxy is to show no signatures (i.e., tidal tails, asymmetries, etc.) of present or past interactions. In the case of NGC 1277, this analysis is complicated due to the presence of two galaxies aligned near our object (Figure 1, left panel). Consequently, in order to study potential faint evidence of interactions, we have subtracted the light contamination from those galaxies. We have modeled and subtracted the light of the two intervening galaxies using the IRAF task ELLIPSE (Jedrzejewski 1987).

The clean image of NGC 1277 is shown in Figure 1, right panel. We see no tidal signatures down to the surface brightness limit of this image ( $26.8 \mathrm{mag} \operatorname{arcsec}^{-2}$; $r$-band). In fact, NGC 1277 looks very regular along its entire structure. A conservative estimate suggests that the above surface brightness limit implies that any potentially "hidden" disrupted satellite around NGC 1277 would contribute less than $2 \%$ to the mass of the galaxy. For this reason, we conclude that NGC 1277 does not present signs of currently (or in its immediate past) accreting external stars.

\subsection{Stellar Mass Density Profile}

The second piece of evidence to suggest that NGC 1277 has the structural characteristics of a primitive massive galaxy is given by its stellar mass density profile. Although our galaxy is clearly elongated, the mass density profiles of the galaxies which we have used in our control samples, both at low and high- $z$ (see next paragraph), were obtained using circular apertures. For this reason, to make a fair comparison with those control galaxies, we have obtained the surface brightness profile of our galaxy using circular apertures. Once we obtained the F625W ( $r$-band) surface brightness profile, we created the stellar mass density profile of the galaxy assuming that there are no stellar population gradients throughout its entire structure. This approximation is reasonable according to the information provided by the deep 


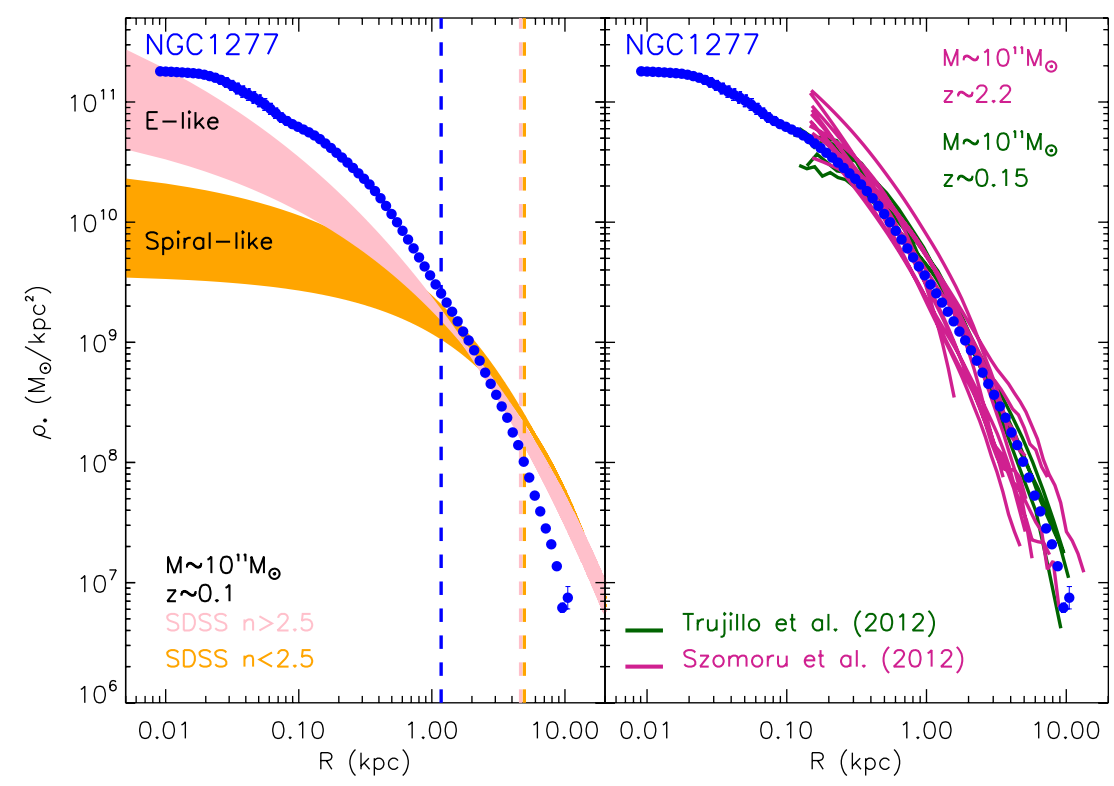

Figure 2. Circularized stellar mass density profile of NGC 1277. The left panel shows the comparison between the stellar mass density profile of NGC 1277 and similar mass but normal-sized SDSS galaxies. The vertical dashed lines indicate the position of the effective radii for NGC 1277 (blue line; $1.2 \mathrm{kpc}$ ) and SDSS massive ellipticals (pink line; $4.7 \mathrm{kpc}$ ) and spirals (orange line; $5 \mathrm{kpc}$ ). The right panel shows the comparison of the stellar mass density profile of NGC $1277 \mathrm{with}$ similar mass compact galaxies at high- $z$ and young compact massive objects at $z \sim 0.15$.

(A color version of this figure is available in the online journal.)

spectra we will show in the next subsection. Using the age and metallicity from our analysis of the galaxy spectra (12 Gyr and 0.2 dex respectively, see below), we have obtained a $\mathrm{M} / \mathrm{L}$ of 4.438 ( $r$-band) which we use to build the stellar mass density profile (see Figure 2).

In the left panel of Figure 2, we show the comparison of the stellar mass density profile of NGC 1277 with the stellar mass density profiles of "normal-sized" massive galaxies from the SDSS survey. To build the stellar mass density profiles of the "normal-sized" galaxies, we took the structural parameters (Sérsic index $n$, effective radius $R_{e}$, and stellar mass $M_{\star}$ ) of all the galaxies in the New York University (NYU) ValueAdded Galaxy Catalog (Blanton et al. 2005) with $0.8<M_{\star}<$ $1.2 \times 10^{11} M_{\odot}$ and $0.08<z<0.12$. To facilitate the comparison with our profiles, we divided the NYU galaxies into two different categories: disk-like $(n<2.5)$ and spheroid-like $(n>2.5)$. We find that the average disk-like massive galaxy within the NYU sample at those redshifts has $M_{\star}=0.92 \times 10^{11} M_{\odot}, n=2.2$, and $R_{e}=5.0 \mathrm{kpc}$. On the other hand, the average spheroid-like object has $M_{\star}=0.95 \times 10^{11} M_{\odot}, n=4.0$, and $R_{e}=4.7 \mathrm{kpc}$. Once we obtained these average galaxy profiles, the representative regions of each galaxy category were build using all the galaxies in the NYU sample within the above stellar mass range and redshift interval, the central stellar mass densities of which were within $68 \%$ of the distribution centered around the mean value.

Neither present-day massive "normal-sized" spiral-like nor elliptical-like galaxies have similar stellar mass density profiles to that shown by NGC 1277. NGC 1277 has a denser profile in the inner $1 \mathrm{kpc}$ (being a factor of two to three denser than the present-day densest elliptical galaxies of the same stellar mass). In the outer region, beyond $4 \mathrm{kpc}$, NGC 1277 is underdense compared to the normal population of massive galaxies. However, when we compare (see Figure 2 right panel) the stellar mass density profile of NGC 1277 with the massive compact galaxies found at high- $z$ (Szomoru et al. 2012) or with the young compact massive galaxies found at $z \sim 0.15$ (Trujillo et al. 2012), the agreement between both profiles is remarkable. We conclude that the detailed structural properties of NGC 1277 are equivalent to those found in the primitive universe for massive galaxies with similar stellar mass.

\subsection{Stellar Population Properties and SFH}

The final evidence for considering that NGC 1277 is a relic galaxy comes from a detailed analysis of its spectra along its major axis (see Figure 3). In the upper panel of this figure, we present the spectra of the galaxy at different radial distances. The depth of our exposure allows us to explore the stellar population properties of our galaxy down to $3 R_{e}$ with an $\mathrm{S} / \mathrm{N}$ above 20 (our limit to ensure that we have sufficient quality to obtain reliable SFHs; Cid Fernandes et al. 2013).

The SFHs of NGC 1277 along its radial distance have been obtained using a full spectral fitting approach. We have employed the extended versions of the MILES stellar population synthesis models (Vazdekis et al. 2010) MIUSCAT (Vazdekis et al. 2012; Ricciardelli et al. 2012) and we have fed the full spectral fitting code STARLIGHT (Cid Fernandes et al. 2005) with them in order to recover the SFH of this galaxy. The models cover a wide range of both ages (0.1-17.8 Gyr) and metallicities ( -1.71 to 0.22$)$ and they also allow for variations on the initial mass function (IMF) slope and shape. For the purpose of this exercise, we have focused on the standard assumption of a Kroupa Universal IMF, although we have also studied the impact on changing the IMF (Ferré-Mateu et al. 2013) according to the velocity dispersion, as recently claimed by several studies (e.g., Ferreras et al. 2013; La Barbera et al. 2013), and we have found that our results remain unaltered. In addition, the same study was carried out with a different spectral fitting code, STECKMAP (Ocvirk et al. 2006), to ensure the robustness of the method, producing similar results.

We therefore derive the mean age and mean metallicity and the SFH for each of the radial binnings out to $3 R_{e}$ (both luminosity and mass-weighted). Figure 3 (lower panels) shows the derived mass-weighted SFH for four of the apertures. It 

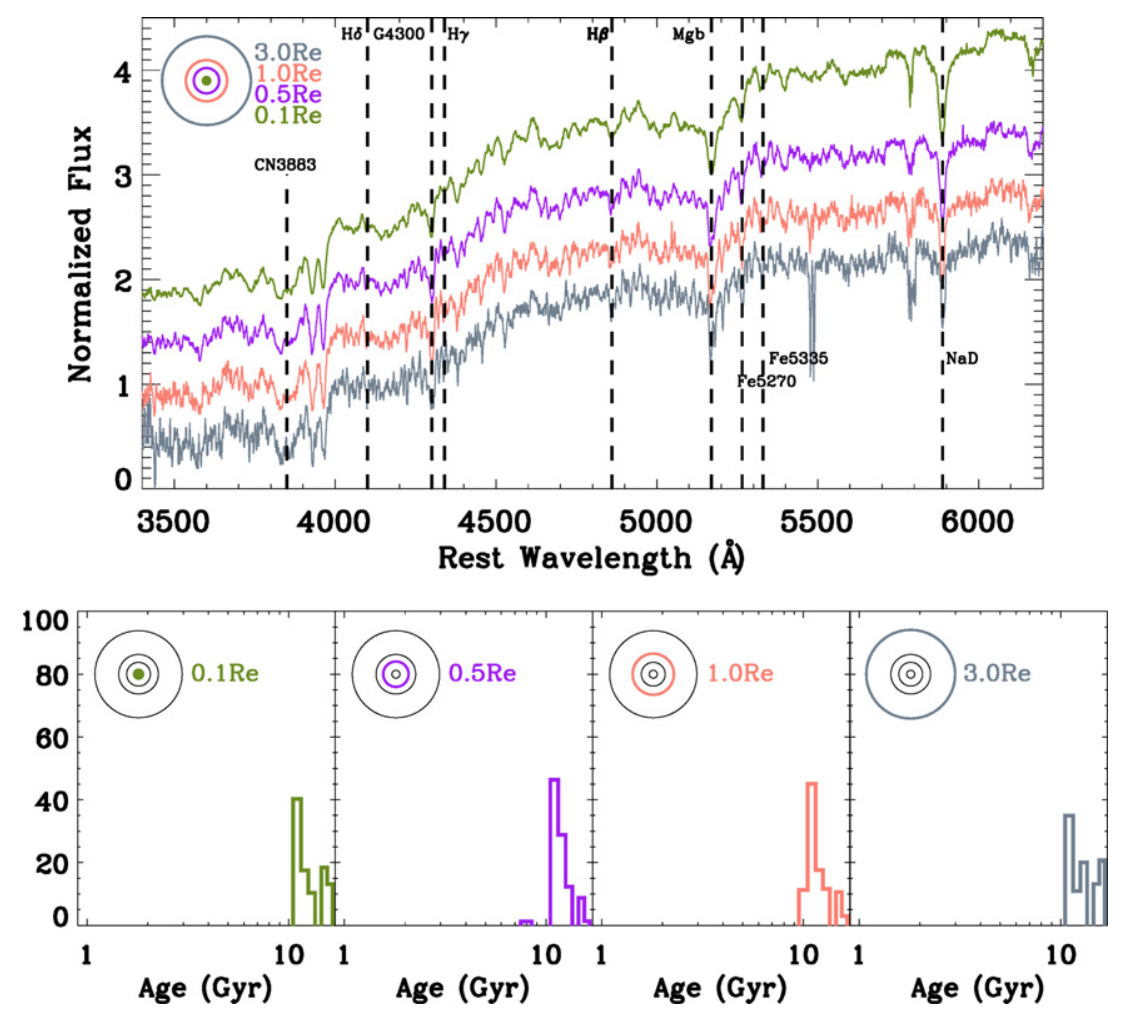

Figure 3. Spectral energy distribution of NGC 1277 at different radial distances. The spectra are shown in arbitrary units, with the flux normalized per unit wavelength and shifted for clarity. The position of several relevant absorption lines are indicated with vertical lines. The bottom panels show the SFHs derived with STARLIGHT at different radial distances. This represents the fraction of mass created at each epoch.

(A color version of this figure is available in the online journal.)

is clear that all of the stars in this object were formed at high-redshift, most of them being older than 10 Gyr. In fact, this remains true even out to large galactocentric distances, as we show in Figure 4, where the radial profiles for the massweighted age (top panel) and metallicity (middle panel) are shown. According to the analysis performed with STARLIGHT, NGC 1277 is an old object $(\sim 12$ Gyr $)$ with a high total metallicity down to at least $3 R_{e}$. This corresponds to a radial distance which encloses $85 \%$ of the total light of this object (Trujillo et al. 2001) assuming a global Sérsic index of $n=$ 2.2 for this galaxy (van den Bosch et al. 2012). In addition to the $\mathrm{SFH}$, age, and metallicity profiles obtained with the full spectral fitting approach, the middle and lower panel in Figure 4 present the radial variation of the metallicity and the $\alpha / \mathrm{Fe}$ parameter, which is a good estimator of how fast a galaxy forms its stars (Worthey et al. 1992) using line strength indices. This metallicity profile has been estimated using a hybrid approach which combines the luminosity weighted age derived from STARLIGHT versus the [MgFe]' index. This metallicity gradient shows similar values in the inner region as the one purely based on STARLIGHT. Both metallicity profiles seems to indicate a smooth decline toward lower metallicities in the outer parts of NGC 1277.

To estimate the $\alpha / \mathrm{Fe}$ gradient, since the MIUSCAT models are scaled solar around the solar metallicity, we employ the approach shown in La Barbera et al. (2013). We have first derived two independent metallicity estimates from the pair of spectral indices $\mathrm{H} \beta-\mathrm{Mgb}_{5177}$ and $\mathrm{H} \beta-\langle\mathrm{Fe}\rangle^{*}$ (a combined $\mathrm{Fe}$ index; González 1993), $\mathrm{Z}_{\mathrm{Mg}}$ and $\mathrm{Z}_{\mathrm{Fe}}$ respectively. The difference between these two metallicities is a good solar proxy $\left[\mathrm{Z}_{\mathrm{Mg}} / \mathrm{Z}_{\mathrm{Fe}}\right]$ and has been shown to tightly correlate with the $\alpha$-enhanced single stellar population models of Thomas et al. (2011). We have found $\left[\mathrm{Z}_{\mathrm{Mg}} / \mathrm{Z}_{\mathrm{Fe}}\right] \sim 0.7$ in our spectra, which translates into $[\alpha / \mathrm{Fe}] \sim 0.4$. Note that an extrapolation has been made when employing the model grid corresponding to the Mgb index (see also Figure 5 in La Barbera et al. 2013), and therefore these values should be taken with some caution. Nonetheless, such very high values indicate that the bulk of the stellar populations of this object, which are very old, was formed in a very short period of time, almost resembling a single-burst event. In fact, according to the calibration provided by de La Rosa et al. (2011, their Equation (2)) between the time needed to form half of the final stellar mass of the galaxy, $\mathrm{T}_{M / 2}$, and $\alpha / \mathrm{Fe}$, NGC 1277 formed its mass in less than a few hundred Myrs. For comparison, a normal-sized massive galaxy with $\alpha / \mathrm{Fe}=$ 0.25 , has $\mathrm{T}_{M / 2}=1.4 \mathrm{Gyr}$. In other words, NGC 1277 seems to have formed its stellar mass much faster than "normal-sized" galaxies with equal mass. Such short time scales suggest star formation rates as high as $\sim 1000 M_{\odot} \mathrm{yr}^{-1}$ for the formation of the bulk of the stars of NGC 1277. These high star formation rates have been measured in massive high- $z$ galaxies (Riechers et al. 2013).

\section{RESULTS AND DISCUSSION}

We have presented three strong pieces of evidences that suggest that NGC 1277 is a relic galaxy. It is massive and compact, with no signature of interactions, it has exactly the same stellar mass density profile as the massive compact galaxies at high- $z$, and finally its stellar populations are compatible with having been formed very fast and more than $10 \mathrm{Gyr}$ ago. Consequently, it is worth exploring its other properties to learn about the 


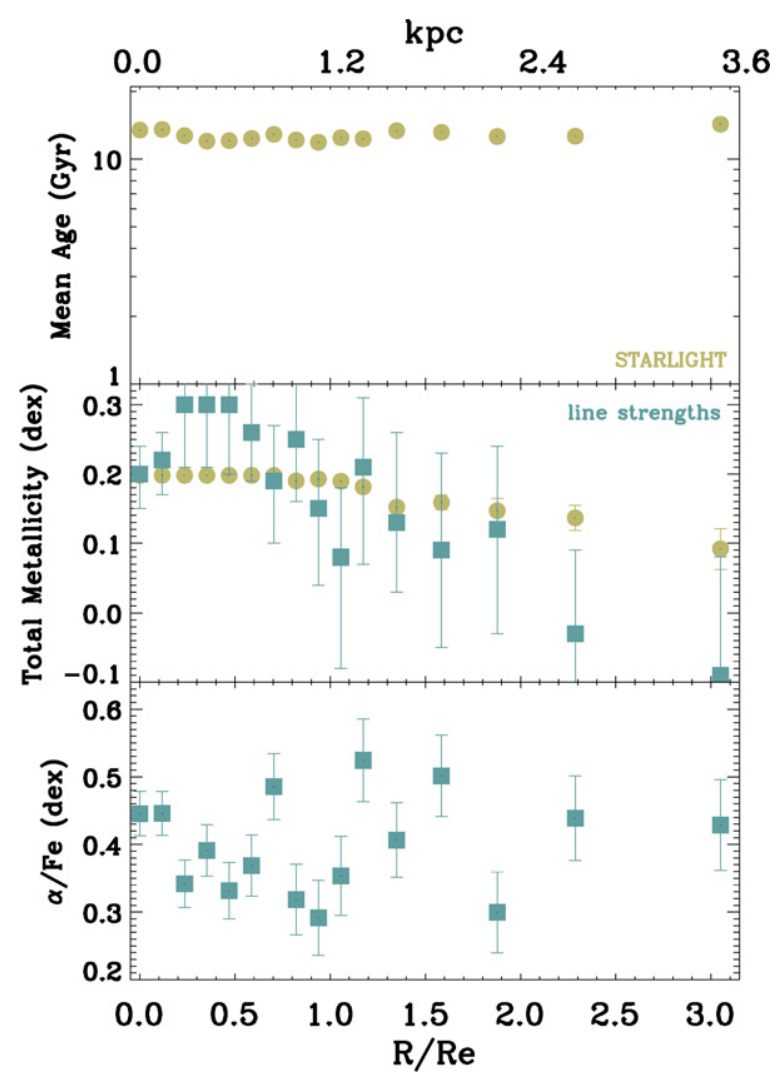

Figure 4. Age, metallicity, and $\alpha / \mathrm{Fe}$ profiles of NGC 1277. The figure shows the mean mass-weighted age and total metallicity derived using STARLIGHT. An independent measure of the metallicity and $\alpha / \mathrm{Fe}$ was obtained from an indices analysis. The profiles show a small change of the stellar population properties of this relic galaxy across its structure out to $3 R_{e}$. It seems that the entire galaxy was formed in a unique, very fast event, which produced the high $\alpha / \mathrm{Fe}$ abundances.

(A color version of this figure is available in the online journal.)

physical conditions at the formation of massive galaxies in the early universe.

No doubt, one of the most remarkable claims about NGC 1277 is the presence of an over-massive blackhole in its center (van den Bosch et al. 2012). The exact mass of this SMBH is controversial, with claims that range from $\sim 2 \times 10^{10} M_{\odot}$ to the more modest (but still a factor of $\sim 4$ larger than the expectation for the mass of this galaxy) values of $\sim 2 \times 10^{9} M_{\odot}$ (Emsellem 2013). One is tempted to claim that the reason why this SMBH is much larger than what it is expected is the absence of further growth in mass of its host galaxy after its initial assembly. In fact, an object with the characteristics of NGC 1277 is expected to have doubled its mass since $z \sim 2$ due to major and minor (dry) merging (van Dokkum et al. 2010). This accreted stellar mass is mainly deposited in the outer region of the galaxy without feeding the central SMBH with new gas. For this reason, if NGC 1277 had followed the normal growth path expected for this type of galaxy, it would have had a more "normal" SMBH. If this picture is correct (see also Kormendy \& Ho 2013), it seems reasonable to suggest that the SMBHs (at least for the most massive galaxies) were formed together with the bulk of the stars of their host galaxies in a very fast collapse at high- $z$. After that, the SMBHs have remained unchanged in mass while the masses of the host galaxies have continued to grow by successive merging. It would be worth exploring whether the peculiar evolutionary path followed by NGC 1277 was dictated by its location in a such rich cluster as Perseus.
The morphology and internal dynamics of NGC 1277 are also interesting. Visually, NGC 1277 has been classified as a peculiar S0 (Corwin et al. 1994). In fact, its elongated shape resembles such a morphology. It is worth noting that high ellipticity is common among massive compact galaxies, both at high- $z$ (e.g., van der Wel et al. 2011; Buitrago et al. 2013) and at $z \sim 0.15$ (Trujillo et al. 2012). Finally, in relation to the dynamics of NGC 1277, it is worth mentioning the high central velocity dispersion $\left(333 \mathrm{~km} \mathrm{~s}^{-1}\right)$ as well as its fast rotation $\left(\sim 300 \mathrm{~km} \mathrm{~s}^{-1}\right)$ measured along its major axis (van den Bosch et al. 2012). To go further into dynamical analysis, and also to better address the morphology of NGC 1277 , is necessary to explore the dynamics of this object with three-dimensional spectroscopy. Currently we can only speculate with the information available along the major axis. If this galaxy was in fact formed in a very fast event, we can consider that the dynamics of its most inner region could resemble the turbulent and chaotic motions of the shocks of enormous cold flows triggering the star formation in its center. Its fast rotation also could be related to the compact structure of NGC 1277. The angular momentum conservation could have transformed an original modest rotational velocity of the infalling gas cloud into the high values we observe now for NGC 1277 outer stars.

Finally, if the theoretical predictions by Quilis \& Trujillo (2013) are correct, one would expect to find only a single relic galaxy every $10^{6} \mathrm{Mpc}^{3}$. It turns out that this number is very close to the volume enclosed by a sphere with a radius of $73 \mathrm{Mpc}$ $\left(\sim 1.6 \times 10^{6} \mathrm{Mpc}^{3}\right)$. This again indicates the unique nature of NGC 1277.

We thank the referee for constructive comments. This work benefited from interesting discussions with Jesús FalcónBarroso and Francesco La Barbera. This article is based on observations made with the WHT operated on the island of La Palma by the Isaac Newton Group of Telescopes in the Spanish Observatorio del Roque de los Muchachos. This research has been supported by the Spanish Ministerio de Economía y Competitividad (MINECO; grants AYA201021322-C03-02 and AYA2009-11137). A.F.M. acknowledges the Japan Society for the Promotion of Science (JSPS) Grant-in-Aid for Scientific Research (KAKENHI) Number 23224005.

\section{REFERENCES}

Blanton, M. R., Schlegel, D. J., Strauss, M. A., et al. 2005, AJ, 129, 2562

Buitrago, F., Trujillo, I., Conselice, C. J., \& Haußler, B. 2013, MNRAS, 428, 1460

Cardiel, N. 1999, PhD thesis, Univ. California, Santa Cruz

Cid Fernandes, R., Gonzalez Delgado, R. M., Garcia Benito, R., et al. 2013, arXiv:1307.0562

Cid Fernandes, R., Mateus, A., Sodré, L., Stasińska, G., \& Gomes, J. M. 2005, MNRAS, 358, 363

Corwin, H. G., Jr., Buta, R. J., \& de Vaucouleurs, G. 1994, AJ, 108, 2128

de La Rosa, I. G., La Barbera, F., Ferreras, I., \& de Carvalho, R. R. 2011, MNRAS, 418, L74

Emsellem, E. 2013, MNRAS, 433, 1862

Ferré-Mateu, A., Vazdekis, A., \& de la Rosa, I. G. 2013, MNRAS, 431, 440 Ferré-Mateu, A., Vazdekis, A., Trujillo, I., et al. 2012, MNRAS, 423, 632

Ferreras, I., La Barbera, F., de la Rosa, I. G., et al. 2013, MNRAS, 429, L15

González, J. J. 1993, PhD thesis, Univ. Complutense de Madrid

Jedrzejewski, R. I. 1987, MNRAS, 226, 747

Kormendy, J., \& Ho, L. C. 2013, ARA\&A, 51, 511

La Barbera, F., Ferreras, I., Vazdekis, A., et al. 2013, MNRAS, 433, 3017

Ocvirk, P., Pichon, C., Lançon, A., \& Thiébaut, E. 2006, MNRAS, 365, 46

Poggianti, B. M., Calvi, R., Bindoni, D., et al. 2013, ApJ, 762, 77

Quilis, V., \& Trujillo, I. 2013, ApJL, 773, L8

Ricciardelli, E., Vazdekis, A., Cenarro, A. J., \& Falcón-Barroso, J. 2012, MNRAS, 424, 172 
Riechers, D. A., Bradford, C. M., Clements, D. L., et al. 2013, Natur, 496, 329 Szomoru, D., Franx, M., \& van Dokkum, P. G. 2012, ApJ, 749, 121

Taylor, E. N., Franx, M., Glazebrook, K., et al. 2010, ApJ, 720, 723

Thomas, D., Maraston, C., \& Johansson, J. 2011, MNRAS, 412, 2183

Trujillo, I., Carrasco, E. R., \& Ferré-Mateu, A. 2012, ApJ, 751, 45

Trujillo, I., Cenarro, A. J., de Lorenzo-Cáceres, A., et al. 2009, ApJL, 692, L118

Trujillo, I., Conselice, C. J., Bundy, K., et al. 2007, MNRAS, 382, 109

Trujillo, I., Graham, A. W., \& Caon, N. 2001, MNRAS, 326, 869

Valentinuzzi, T., Fritz, J., Poggianti, B. M., et al. 2010, ApJ, 712, 226 van den Bosch, R. C. E., Gebhardt, K., Gültekin, K., et al. 2012, Natur, 491, 729

van der Wel, A., Rix, H.-W., Wuyts, S., et al. 2011, ApJ, 730, 38

van Dokkum, P. G., Whitaker, K. E., Brammer, G., et al. 2010, ApJ, 709, 1018 Vazdekis, A., Peletier, R. F., Beckman, J. E., \& Casuso, E. 1997, ApJS, 111,203

Vazdekis, A., Ricciardelli, E., Cenarro, A. J., et al. 2012, MNRAS, 424, 157

Vazdekis, A., Sánchez-Blázquez, P., Falcón-Barroso, J., et al. 2010, MNRAS, 404, 1639

Worthey, G., Faber, S. M., \& Gonzalez, J. J. 1992, ApJ, 398, 69 